\title{
Cognitive-behavioural therapy for non-motor symptoms of Parkinson's disease: a clinical review
}

\author{
Ivan Koychev, ${ }^{1}$ David Okai ${ }^{2}$
}

\begin{abstract}
${ }^{1}$ Department of Psychiatry, University of Oxford, Warneford Hospital, Oxford, UK; ${ }^{2}$ Psychological Medicine Service, Oxford University Hospitals NHS Foundation Trust, Oxford, UK

Correspondence to Dr Ivan Koychev, Department of Psychiatry, University of Oxford, Warneford Hospital, Oxford OX3 7JX, UK; ivan.koychev@doctors.org.uk
\end{abstract}

\begin{abstract}
Neuropsychiatric symptoms are common in Parkinson's disease (PD) and have a disproportionate impact on quality of life and carer burden. Pharmacological treatment is the main approach in dealing with these symptoms, but it is limited by variable efficacy and risk of drug interactions. Non-pharmacological approaches using the cognitive-behavioural therapy (CBT) model are viable alternatives and in this review paper we summarise the evidence of CBT for three of the most common psychiatric manifestations of PD: depression and anxiety, impulse-control disorders and insomnia. Most studies modified the usual CBT format to include modules accounting for problems specific to PD: activity scheduling around motoric function, motor symptoms as triggers of anxiety, fear of falling and preparation for disease progression as well as accommodation of materials for suspected executive dysfunction. We found a growing evidence base that CBT (modified to account for PD-specific problems) is effective in the treatment of PD psychiatric symptoms. Where controlled study design was used, moderate effect sizes are reported for the efficacy of CBT for depression, including with distance administration of CBT. The effects were sustained during follow-up which was between 1 and 6 months. In addition, there are some initial data on the effects of CBT on impulse-control disorders and insomnia. The studies were limited by their small and potentially unrepresentative samples and the quality of sample reporting (eg, concomitant antidepressant and dopaminergic therapy use). Additional welldesigned and adequately powered studies are required to determine the utility of CBT in PD.
\end{abstract}

\section{INTRODUCTION}

Parkinson's disease (PD) is classically defined by a triad of motor symptoms (tremor, rigidity and bradykinesia). It is, however, increasingly conceptualised as a neuropsychiatric disorder ${ }^{1}$ in view of the high prevalence of psychiatric features, including depression, impulse control disorders (ICD) and sleep difficulties. ${ }^{2}$ These non-motor complications are better predictors of disability, distress and rates to institutionalisation than the motor symptoms. ${ }^{3}$ Of the non-motor complications, mixed depression and anxiety is the most common psychiatric comorbidity, affecting $\sim 50 \%$ of PD patients, significantly more common that rates in the general population. ${ }^{4}$ Depression and anxiety are the strongest predictors of poorer quality of life, ${ }^{5}$ even in the advanced stages of the illness where the motor features have progressed fully. ${ }^{6}$ ICD are characterised by repetitive, reward-based behaviours, linked to the dopaminergic medication used to treat the motor symptoms. They affect $\sim 14 \%$ of all PD patients ${ }^{7}$ and are associated with psychiatric comorbidity and high carer burden. ${ }^{8}$ Sleep disturbance is another neuropsychiatric symptom that affects up to $60 \%$ of all PD patients. ${ }^{9}$ It is associated with somnolence ${ }^{10}$ and impairment in attention and executive function. ${ }^{11}$ On the basis of their impact on quality of life and outcome, the recognition and treatment of neuropsychiatric symptoms in $P D$ is increasingly viewed as a priority in PD management. ${ }^{1}$

A pharmacological approach is often adopted in the management of depression, ICD and sleep disturbance in PD. In the case of depression and anxiety, the efficacy of medication was found to be of moderate effect size but statistically non-significant in a pooled analysis. ${ }^{12}$ Other issues with antidepressants include concerns over polypharmacy ${ }^{13}$. A further complicating factor is that comorbidity with anxiety predicts lower response rate to antidepressants in PD patients. ${ }^{14}$ The usual management of ICDs relies on the withdrawal or reduction in dose of the dopamine replacement therapies. However, many patients fail to tolerate this on account of worsening control of PD symptoms, while in some the symptoms persist despite medication change. ${ }^{15}$ While there are a variety psychopharmacological treatment options for insomnia, ${ }^{16}$ their efficacy in PD is limited by its potential genesis: degeneration of sleep regulatory nervous system areas. ${ }^{17}$ Also, PD patients mainly have difficulties in sleep maintenance rather than initiation ${ }^{18}$ - this is an issue as most pharmacological agents promote sleep initiation. Therefore, while pharmacological treatment of neuropsychiatric features in PD is useful and well-established, it is often limited in terms of efficacy and practicality.

The current unmet need in terms of PD neuropsychiatric therapy has led to the development of non-pharmacological approaches. The most well studied method is cognitive-behavioural therapy (CBT) - this is a brief (usually 10-12-week) talking therapy that focuses on current behaviours and problems. The underlying assumption is that when in a state of psychological distress an individual's interpretation of everyday situations is skewed which has a negative impact on the actions they take as a result. Exploring the nature of response to individual everyday experiences, therefore, becomes the major focus of assessment and treatment. CBT aims to help people identify and subsequently modify unhelpful thoughts, and change global behaviours that serve to maintain and reinforce distorted thinking. Therefore, treatment becomes a nondidactic process of self-discovery for the patient, facilitated by the therapist, allowing opportunity for new adaptive learning and change. In other words, the patient unlearns unhelpful strategies replacing them with more adaptive behaviours. CBT has a broad evidence base in the treatment of psychiatric disorders in the general population-depression and anxiety, ${ }^{19}$ obsessive-compulsive, ${ }^{20}$ eating $^{21}$ and Tourette's disorders. $^{22}$ Importantly, CBT has also been shown to be efficacious in the treatment of anxiety and depression in the context of chronic neurological $^{23}$ and other physically disabling conditions. ${ }^{24}$

In this review paper, we aimed to provide an up-to-date summary of the evidence of psychological therapy efficacy for three of the main neuropsychiatric manifestations of PD: depression and anxiety, insomnia and impulse control disorder.

\section{METHODS}

We searched Ovid and PubMed records using search terms: 'Cognitive-Behavioural Therapy', 'Behavioural' or 'Psychological therapy' or 'CBT' and 'Parkinson's Disease' or 'PD'. Abstracts of identified articles in English were studied to select those studies that involved an efficacy evaluation or case series report of a psychological therapy for one or more of the three target symptom domains (depression and/or anxiety, 
insomnia and impulse control disorder). In addition, we included reports that were not revealed through our search methodology but were referenced in reports we identified through our original search strategy.

\section{RESULTS}

\section{Evidence for individual psychotherapy for depression and anxiety}

We identified five controlled studies which reported effects of individual (ie, not group-based) psychological intervention on depression and/or anxiety either as primary or secondary measures. ${ }^{25-29}$ Table 1 presents an overview of the findings. In addition, there were four uncontrolled studies and two case series.

The numbers in the controlled studies ranged from 10 to 80 patients who had disease duration of between 5 and 10 years. The CBT courses consisted of 6-12 weekly sessions and modified the typical CBT regime to include a stronger emphasis on relaxation modules, behavioural activation, problem-solving and psychoeducation. ${ }^{25} 2729$ In addition, several studies included modules specific to PD: activity scheduling around on-off effects, motor symptoms as triggers of anxiety, fear of falling and preparation for disease progression. ${ }^{27} 29$ In some instances, there were further modifications to the CBT protocol to accommodate the executive and somatic features of PD: less written material provided to patients, unlimited breaks during session to allow patients to attend to their needs. ${ }^{29}$ CBT were delivered by psychologists as well as in some cases by master students supervised by the main investigator. ${ }^{29}$ The control groups consisted of treatment as usual or waiting lists. The larger studies included parallel carer sessions with the aim of consolidating learning given likely executive dysfunction. ${ }^{25} 27$ All but one study ${ }^{28}$ reported that CBT is substantially beneficial for anxiety and depressive symptoms as recorded through self-reported scales (see table 1 for outcome measures and effect sizes where reported). The effect was generally evident immediately at trial end as well as at follow-up where this had been done (between 1 and 6 months). CBT had either lesser or no effect on broader measures of functioning as well as carer burden (see table 1 for more details). The one study that did not find an effect of CBT on depression had the smallest sample size ( $n=6$ for the CBT group) and was designed to assess the effect of CBT for insomnia. ${ }^{28}$

The largest of the studies ${ }^{25}$ recruited $80 \mathrm{PD}$ patients (mean age $64 \pm 10$ years, disease duration $7 \pm 6$ years) with a DSM-IV diagnosis of depressive disorder. The participants were randomised to either CBT plus educational programme for caregivers $(n=41)$ or clinical monitoring $(n=39)$. The CBT course consisted of 10 weekly sessions and had stronger emphasis on behavioural (behavioural activation) and anxiety management (relaxation training, worry control) techniques as well as sleep hygiene than usual CBT protocols. Caregivers were given 4 weekly educational sessions to help facilitate home-based CBT techniques practice (eg, help patients identify negative thoughts, guidance in assisting with completion of therapy goals). The authors reported a large effect on the primary outcome measure of depression (HAM-D) both at intervention end (Cohen's $d=1.59$ ) and effect was sustained at 4-week follow-up. There was also significant improvement in several secondary outcome measures - anxiety (HAM-A score Cohen's $d=0.98$ ), positive reframing (COPE reframing subscale Cohen's $d=0.8$ ), social functioning (Medical Outcomes Study Short-Form Health Survey score Cohen's $d=0.8$ ) and severity of motor symptoms (UPDRS score Cohen's $d=0.4)$. There was no effect on sleep, inferences, physical disability, social support or carer burden. The study is limited by the large concomitant use of antidepressant treatment $154 \%$ of the whole sample) but their doses had to have been stable for at least 6 weeks prior to study start.

The evidence for CBT efficacy in the treatment of depression and anxiety is supported by four uncontrolled studies ${ }^{30-33}$ and two case series. $^{34} 35$ The uncontrolled study recruited between 4 and 19 patients of similar age and disease duration to the controlled studies (table 1). Therapy duration ranged from 6 to 14 weeks and some studies adapted its content to limitations and problems specific to PD patients. ${ }^{33}$ The uncontrolled studies also reported significant improvement in depressive and anxiety at therapy end and follow-up (two studies followed participants at 4 weeks ${ }^{30} 32$ and one at 24 weeks ${ }^{33}$ ). The two case series (three and five patients) also reported significant improvement for depressive symptoms, ${ }^{34}{ }^{35}$ particularly for those with more severe depression at baseline. ${ }^{34}$

\section{Evidence for distance CBT for depression and anxiety}

Despite the good evidence for the efficacy of CBT in treating depression in $\mathrm{PD}$, its large-scale implementation is limited by practical considerations: PD patients may have difficulty in attending weekly sessions on accounts of their motor disability and reliance on carers for transportation. We identified three studies that employed either distance CBT using telephone ${ }^{36} 37$ or internet methods. ${ }^{38}$ The three studies included samples of between 9 and 21 patients of comparable age and disease duration to the individual CBT studies (mean age of 66 years and disease duration of $\sim 7$ years). Duration of therapy was 9-12 weeks and the programmes involved psychoeducation, relaxation training, activity scheduling and sleep management. In the most comprehensive intervention, Dobkin et $a^{36}$ sent participants a session-by-session manual to prepare them for each call and organised telephone-based education sessions for the carers. Telephone-based CBT was associated with significant improvement in depressive scores at intervention end ${ }^{30}{ }^{37}$ and 4 -week follow-up ${ }^{30}$ but there was no beneficial effect on quality of life. A follow-up analysis of the Dobkin ${ }^{39}$ study showed that baseline verbal and working memory predicted improvement in depression following the 10-week telephone CBT. Internet-based CBT was associated with significant improvement in depressive but not anxiety scores at therapy end. ${ }^{38}$ The authors noted that relative to a larger study using internet CBT in non-PD patients ${ }^{40} \mathrm{PD}$ patients were considerably less active in contacting their therapist and completing assignments and also had lower degree of satisfaction with the therapy.

\section{CBT for carers}

PD is associated with major carer burden (coping with non-motor symptoms; responsibility for mobility, financial and medical affairs) which can lead to loss of quality of life through restriction of other areas of the carer's function. ${ }^{41}$ The CBT model has been used to provide support for carers of PD patients. One group led on the creation of a standardised education programme for patients with PD and their carers. ${ }^{42-44}$ The format involved 8 weekly group sessions of 90 min duration and was implemented across a number of European sites. The content was the same for patients and carers and included psychoeducation sessions, as well as core CBT modules such as relaxation training, cognitive restructuring and situational behaviour analysis. The final sample consisted of 137 carers (they were not selected on the basis of psychological need) and 151 patients. ${ }^{42} 43$ The studies found that the intervention was associated with high degree of acceptability to carers and patients and there was improvement in the psychosocial burden experienced by both groups. Depression scores improved post session (determined using a visual analogue scale) but there was no significant change in depressive self-reported scores at the end of the intervention (there was no follow-up data). A different research group targeted carers experiencing distress (based on General Health Questionnaire 28 $\left(\mathrm{GHO}-28^{45}\right)$ score) and randomised them to CBT $(n=15)$ or treatment $(n=15)$ as usual. ${ }^{46}$ The CBT course lasted for a mean of 15 sessions and followed a classical CBT module structure (challenging negative thoughts and feelings, maladaptive rules; relaxation training; pleasant activity scheduling) plus psychoeducation on the nature of PD and the 
Table 1 Study characteristics

\begin{tabular}{|c|c|c|c|c|}
\hline Study & $\begin{array}{l}\text { Participants and primary } \\
\text { outcome }\end{array}$ & Design & $\begin{array}{l}\text { Demographics of } \\
\text { treatment groups } \\
\text { (mean years, SD) }\end{array}$ & Outcome \\
\hline \multicolumn{5}{|c|}{ Depression and anxiety (individual CBT) } \\
\hline $\begin{array}{l}\text { Cole and } \\
\text { Vaughan }(2005)^{34}\end{array}$ & $\begin{array}{l}5 \text { PD } \\
\text { Primary outcome: GDS }\end{array}$ & $\begin{array}{l}\text { Individual CBT case series } \\
\text { Duration: } 7 \text { weeks; Follow-up: } 8 \text { weeks }\end{array}$ & $\begin{array}{l}\text { Age } 73.6 \pm 13.4 \\
\text { Disease duration } 3.3 \\
\pm 1.9\end{array}$ & $\begin{array}{l}\text { Improvement in mood symptoms in four of five } \\
\text { patients post treatment but sustained effect at } \\
1 \text { month in two of five patients. Significant medical } \\
\text { illness, lower baseline level of depressive symptoms } \\
\text { and cognitive impairment were significant moderators } \\
\text { of long-term gains. CBT was not associated with } \\
\text { change to quality of life. }\end{array}$ \\
\hline $\begin{array}{l}\text { Dobkin et al } \\
(2006)^{35}\end{array}$ & $\begin{array}{l}\text { Three PD and three carers } \\
\text { Primary outcome: HAM-D }\end{array}$ & $\begin{array}{l}\text { Individual CBT and caregiver case } \\
\text { series } \\
\text { Duration: not given; follow-up: } 4 \text { weeks }\end{array}$ & $\begin{array}{l}\text { Age } 62.7 \pm 12.2 \\
\text { Disease duration } 5 \pm 4\end{array}$ & $\begin{array}{l}\text { Reduction in depressive symptoms during intervention } \\
\text { and } 1 \text { month follow-up. There was also a decrease in } \\
\text { negative thoughts and increase in perceived support. } \\
\text { Minimal change to anxiety symptoms. }\end{array}$ \\
\hline $\begin{array}{l}\text { Dobkin et al } \\
(2007)^{30}\end{array}$ & $\begin{array}{l}15 \text { PD and } 15 \text { carers } \\
\text { Primary outcome: HAM-D }\end{array}$ & $\begin{array}{l}\text { Uncontrolled } 10-14-\text { week CBT plus } \\
\text { educational programme for caregivers } \\
\text { Duration: } 10-14 \text { weeks; follow-up: } \\
4 \text { weeks }\end{array}$ & $\begin{array}{l}\text { Age } 64.2 \pm 9.6 \\
\text { Disease duration } 5.1 \\
\pm 3.2\end{array}$ & $\begin{array}{l}\text { Decrease in depression with } 80 \% \text { being treatment } \\
\text { responders. There was also decrease in negative } \\
\text { inferences, increased perception of social support but } \\
\text { non-significant improvement in anxiety. Depression } \\
\text { effect maintained at } 4 \text { months. }\end{array}$ \\
\hline $\begin{array}{l}\text { Dobkin et al } \\
(2011)^{25}\end{array}$ & $\begin{array}{l}80 \text { PD } \\
\text { Primary outcome: HAM-D }\end{array}$ & $\begin{array}{l}\text { Controlled CBT plus educational } \\
\text { programme for caregivers ( } n=41) \text { vs } \\
\text { clinical monitoring }(n=39)\end{array}$ & $\begin{array}{l}\text { Age } 63.7 \pm 9.9 \\
\text { Disease duration } 6.5 \\
\pm 5.5\end{array}$ & $\begin{array}{l}\text { Significant reduction in depression scores; } \\
\text { Improvement for anxiety }(d=1.19) \text {, stress }(d=1.10) \\
\text { social functioning, UPDRS ( week } 10 \text { and 14). }\end{array}$ \\
\hline
\end{tabular}

\begin{tabular}{|c|c|c|}
\hline Dreissig $(1999)^{26}$ & $\begin{array}{l}79 \text { PD } \\
\text { Primary outcome: } \\
\text { PROGRESSOR } 230\end{array}$ & $\begin{array}{l}\text { Controlled self-help and CBT }(n=9) \text { vs } \\
\text { treatment at usual ( } n=70) \\
\text { Duration: } 12 \text { weeks ( } 6 \text { sessions); } \\
\text { Follow-up: none }\end{array}$ \\
\hline $\begin{array}{l}\text { Farabaugh et al } \\
(2010)^{31}\end{array}$ & $\begin{array}{l}8 \text { PD } \\
\text { Primary outcome: HAM-D }\end{array}$ & $\begin{array}{l}\text { Uncontrolled CBT case series } \\
\text { Duration: } 12 \text { weeks; follow-up: none }\end{array}$ \\
\hline
\end{tabular}

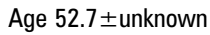
Disease duration 8.6

Improvement in anxiety and hopelessness levels.

土unknown

Age $63.6 \pm 7.5$

Disease duration unknown

$57 \%$ remitted at study end (defined as HAM-D $<7$ ) with a significant reduction in HAM-D scores for the group. HAM-D related to frequency and degree of negative thoughts. There was no main effect of therapy on scales of perceived stress and automatic thoughts.

Uncontrolled CBT case series $\quad$ Age $65.3 \pm 11$

Duration: 8 weeks; follow-up: 12 weeks Disease duration 4.8

3 of the 4 patients improved in terms of depression at 1 month follow-up and 2 of 4 at intervention end. No clinically significant improvement in anxiety scores. Significant improvement of depression at study end (HAM-D score, Hedges' $g=-1.02$ ) with the effects being maintained at 3-month follow-up.

Significant improvement in anxiety and depression scores post treatment. Improvement sustained at 6 months (Cohen's $d=0.94$ to $d=2.26$ ). No change to quality of life. Significant reduction in frequency of depressive (Cohen's $d=1.26$ ) and anxiety (Cohen's $\mathrm{d}=0.92$ ) thoughts.

Depression and anxiety (distance CBT)

$\begin{array}{llll}\text { Dobkin et al } & 21 \mathrm{PD} & \text { Uncontrolled telephone CBT plus } & \text { Age } 65.9 \pm 9.4 \\ (2011)^{36} & \text { Primary outcome: HAM-D } & \text { caregiver educational sessions } & \text { Disease duration } 7.5 \\ & & \text { Duration: } 10 \text { weeks; follow-up: } 4 \text { weeks } & \pm 9.4\end{array}$

Significant improvement in depression with $62 \%$ meeting criteria for response at treatment end and $52 \%$ at 4 -week follow-up. Gains in depression severity (BDI score), negative thoughts and coping. Treatment gains at 5-weeks post intervention start and maintained at 4-week follow-up. No significant effects on quality of life, problem-focused coping, sleep, social support or carer burden.

\begin{tabular}{|c|c|c|}
\hline $\begin{array}{l}\text { Kraepelien et al } \\
(2015)^{38}\end{array}$ & $\begin{array}{l}9 \text { PD } \\
\text { Primary outcome: HADS }\end{array}$ & $\begin{array}{l}\text { Uncontrolled internet-based CBT } \\
\text { Duration: } 12 \text { weeks; follow-up: None }\end{array}$ \\
\hline $\begin{array}{l}\text { Veazey et al } \\
(2009)^{37}\end{array}$ & $\begin{array}{l}10 \text { PD } \\
\text { Primary outcomes: } \mathrm{PHQ}-9 \\
\text { and BAI }\end{array}$ & $\begin{array}{l}\text { Controlled telephone CBT }(n=5) \text { vs } \\
\text { support group }(n=5) \\
\text { Duration: } 8 \text { weeks; follow-up: } 4 \text { weeks }\end{array}$ \\
\hline \multicolumn{3}{|c|}{ mpulse control disorder } \\
\hline $\begin{array}{l}\text { Okai et al } \\
(2013)^{27}\end{array}$ & $\begin{array}{l}45 \text { PD } \\
\text { Primary outcomes: CGI, } \\
\mathrm{NPI} \text { and carer burden }\end{array}$ & $\begin{array}{l}\text { Controlled face-to-face CBT ( } n=28 \text { ) vs } \\
\text { waitlist ( } n=17) \\
\text { Duration: } 12 \text { weeks; follow-up: } \\
12 \text { weeks }\end{array}$ \\
\hline
\end{tabular}

Age $66 \pm 11.6$

Disease duration 8.1 $\pm 3.9$

Age $66 \pm 9.9$

Disease duration unknown

Age $59.3 \pm 8.1$ Disease duration 10.5 $\pm 6.0$
Significant improvement in depression and anxiety scores (HADS). No significant change in MADRS-S, insomnia, quality of life or non-motor symptoms. Moderate degree of satisfaction with therapy.

Improvement in anxiety and depression scores for groups vs baseline. Quality of life reduced in CBT group at 4-week follow-up.

Significant improvement in CGI and NPI. $44 \%$ of treatment group did not meet ICD criteria at 12-week follow-up. No significant improvement in carer burden or distress. Improvement in work and social adjustment, anxiety and depression. 
Table 1 Continued

\begin{tabular}{|c|c|c|c|c|}
\hline Study & $\begin{array}{l}\text { Participants and primary } \\
\text { outcome }\end{array}$ & Design & $\begin{array}{l}\text { Demographics of } \\
\text { treatment groups } \\
\text { (mean years, SD) }\end{array}$ & Outcome \\
\hline $\begin{array}{l}\text { Jimenez-Murcia } \\
\text { et al }(2012)^{47}\end{array}$ & $\begin{array}{l}15 \text { PD and } 45 \text { non-PD } \\
\text { pathological gamblers } \\
\text { Primary outcomes: SOGS }\end{array}$ & $\begin{array}{l}\text { Retrospective controlled CBT } \\
\text { Duration: } 8 \text { weeks; follow-up: }\end{array}$ & $\begin{array}{l}\text { Age } 62.7 \pm 8.5 \\
\text { Disease duration } \\
\text { unknown }\end{array}$ & $\begin{array}{l}\text { The authors found higher rate of relapse ( } 25 \% \text { vs } 11 \% \text { ) } \\
\text { and drop-out from therapy ( } 29 \% \text { vs } 9 \% \text { ) for the PD } \\
\text { relative non-PD pathological gamblers. However, this } \\
\text { difference did not reach statistical significance. }\end{array}$ \\
\hline
\end{tabular}

Insomnia

Rios Romenets

et al $(2013)^{28}$

18 PD

Primary outcomes: SCOPA and ISI
Controlled face-to-face of group CBT

plus light therapy $(n=6)$ vs Doxepine

$10 \mathrm{mg}(\mathrm{n}=6)$ vs placebo light therapy

$(n=6)$

Duration: 6 weeks; follow-up: none

Uncontrolled telephone and

face-to-face CBT

Duration: 4 weeks; follow-up: 12 weeks

Group educational programme with

CBT elements for patients and

caregivers

Duration: 8 weeks; follow-up: none

Group educational programme with

CBT elements for patients

Duration: 8 weeks; follow-up: none

Questionnaire on the acceptability of

programme

Secker and

Brown $(2005)^{46}$

30 carers

Primary outcome: GHQ-28

Controlled study of session CBT

$(n=15)$ vs treatment as usual $(n=15)$

Duration: 12-14 weeks; follow-up:

12 weeks

Group educational programme with

Simons et al $2006^{44}$

CBT elements for patients and

caregivers

Duration: 8 weeks; follow-up: none
Age $64.5 \pm 16.3$

Disease duration 5.2

$\pm 1.8$

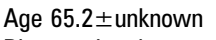
Disease duration

unknown

Age $64.4 \pm 9.2$

Disease duration 6.5 $\pm 4.3$

Age $64.4 \pm 9.2$

Disease duration unknown

Age $59.1 \pm 12.2$

Disease duration: not applicable
Significant improvement in insomnia severity (ISI) but not SCOPA sleep score (doxepine was better than placebo for both). Examiner-rated but not participant-rated CGI improved. No change to daytime sleepiness, BDI, sleep hygiene, dysfunctional sleep beliefs or cognition. No improvement in depression scores with CBT.

Improvement in PDSS and sleep diary measures (onset latency, wake after sleep onset, total sleep time, time in bed and sleep efficiency).

Patients: improvement in psychosocial burden and visual-analogue scale depression score but no improvement in quality of life or self-reported depression. Carers: improvement in psychosocial burden and visual-analogue scale mood scores but no change to health state and self-reported depression.

$67-80 \%$ felt intervention was appropriate and fulfilled expectations. Significant improvement in mood (visual-analogue scale) and psychosocial problems. No change to quality of life and self-rating depression score

Reduction of psychological distress (total GHQ-28 scores) for the treatment group at intervention end. The CBT group also had a decrease in somatic, anxiety, insomnia, social dysfunction but not depression GHQ-28 scores (as well as GDS scores). Effect was maintained at 12-week follow-up.

Age $65 \pm 7.3$

Disease duration 7.6 $\pm 5.0$

Patients and carers approved of the programme and improved in terms of visual-analogue mood scores. No significant differences for carers' quality of life, psychosocial problems, self-reported depression.

BAI, Beck Anxiety Inventory; BDI, Beck Depression Inventory; BELA-A-K, Belastungsfragenbogen Parkinson Angehörigen kurzversion; CGI, Clinical Global Impression; DASS-21, Depression, Anxiety and Stress Scale-21; GDS, Geriatric Depression Scale; GHO-28, General Health Questionnaire-28 items; HADS, Hospital Anxiety and Depression scale; HAM-D, Hamilton Depression Scale; ISI, Insomnia Severity Index; MADRS-S, Montgomery Asberg Depression Rating Scale-Self rating version; NPI, Neuropsychiatric Inventory; PDSS, Parkinson's Disease Sleep Scale; PHO-,9Patient Health Questionnaire-9; PROGRESSOR 230, The Psychological Profile Questionnaire; SCOPA-S, Scales for Outcomes in PD-Sleep; SOGS, South Oaks Gambling Screen; STAI-S, State subscale of State and Trait Anxiety Inventory.

available healthcare and social support for PD. The authors found that the CBT arm had a significant improvement in GHO-28 scores that remained at months 3 and 6 follow-ups. There was an associated improved in anxiety, insomnia and somatic symptoms but not depressive scores.

\section{CBT for impulse-control disorder in PD}

Two studies to date have evaluated the efficacy of CBT in the treatment of ICD in $\mathrm{PD}^{27}{ }^{47}$ In the larger study, the authors recruited $45 \mathrm{PD}$ patients, randomising 28 of them to a modified CBT programme (mean age $59 \pm 8$ years, disease duration $11 \pm 6$ years) and 17 to a control waiting list. The CBT intervention consisted of 12 sessions (mostly at patient's homes) and the part relevant to ICD included psychoeducation (focusing on ICD but also on executive dysfunction in PD), motivational interviewing to identify where the patient was in terms of the cycle of change, monitoring of behaviour, problem solving and pleasant activity scheduling. The CBT for patients was augmented with a 4-week carer intervention that aimed at psychoeducation, supporting collaborative problem-solving and recognising organic personality change. The study found reduction in the global level of ICD symptom severity, their frequency and impact. At the 6-month follow-up, $44 \%$ of the treatment group no longer met ICD criteria compared with $29 \%$ of the waiting list group. While there was no significant benefit to carer burden or distress, carers did improve in terms of their levels of anxiety and depression. A smaller, retrospective study reported on the efficacy of CBT in treating pathological gambling in PD $(n=15)$ versus non-PD $(n=45)$ patients. ${ }^{47}$ The CBT schedule was adapted in the PD group to focus on PD psychoeducation, planning of alternative gratifying activities leg, interactions between gambling and timing of dopaminergic drug therapy), coping with other impulse control behaviours. The investigators offered 16 weekly sessions. The authors did not find a difference between the PD and non-PD patients in terms of their treatment response (measured using South Oaks Gambling Screen ${ }^{48}$ ) but there was an indication of higher relapse and therapy drop-out rates among the PD patients. The study was limited by its retrospective nature, convenience of sample (clinic attendees) and lack of concomitant medication reporting.

\section{CBT for insomnia in PD}

Two studies evaluated CBT targeting insomnia in PD. ${ }^{28} 49$ The first study involved 6 weekly face-to-face CBT sessions plus light therapy compared to doxepin and placebo light therapy (each group included 6 patients, mean age 65 with 5 years disease duration). ${ }^{28}$ The second 
study evaluated four-session telephone-based CBT in 22 patients (mean age 65 , most participants had a disease duration of $1-5$ years). ${ }^{49}$ Neither study reported modifications of classical CBT protocols for insomnia. The individual CBT intervention was reportedly beneficial in terms in the sleep quality index relative to doxepine and placebo ${ }^{28}$ but there was no effect on day time sleepiness. The telephone-based intervention reported improvement on total sleep duration, sleep-onset latency and sleep efficiency posttreatment and at 3-month follow-up. ${ }^{49}$

\section{DISCUSSION}

The aim of this review was to evaluate the level of evidence for cognitive-behavioural therapy for three non-motor symptoms in PD: depression and anxiety, ICD and insomnia. We identified a range of controlled, uncontrolled and case series evaluating CBT for depression and anxiety in PD. CBT protocols were frequently modified to account for psychological problems that are more prevalent in PD_-anxiety modules were prioritised with a focus on relaxation techniques, recognising motor symptoms as triggers of anxiety and the highly prevalent fear of falling. Loss of motivation is also highly prevalent leading to a number of CBT protocols, including behavioural activation modules with emphasis on activity scheduling around on/off periods. Some authors accounted for the potential executive dysfunction in PD and amended their CBT materials to ease comprehension. The largest study offered supplemental caregiver educational programmes which was intended to reinforce learning in the patients' environment given their expected executive and memory dysfunction.

Overall, there was consistent evidence for moderate improvement in self-reported depressive and anxiety scores post-treatment and at follow-up assessments of up to 3 months. There are a number of potential mechanisms for this improvement, including improved insight and self-reflection. Most protocols focused on self-monitoring and the beneficial effect could thus be due to improved ability to identify triggers of low mood or anxiety symptoms. Furthermore, the CBT model focuses on improving patients' problem-solving and coping with stress skills. Also, social isolation is a significant factor in patients with PD on accounts of physical disability or perceived stigma. The psychological intervention therefore could have been beneficial through reduction of social isolation. In addition to these considerations, it is likely that the adaptations of the CBT protocol to the requirements for PD were significant factors. PD features a number of severe physical symptoms that can precipitate or perpetuate affective or anxiety symptoms-adapted protocols thus dedicated time to the importance of motor symptoms as triggers for anxiety, fears of falling and preparation for disease progression. It is also likely that the efficacy of CBT was enhanced where its procedures were adapted to account for the practical limitations of $\mathrm{PD}$, for example, organising activity scheduling around on-off effects, accommodating the anticipated executive and somatic features of PD by focusing less on written material provided to patients and providing unlimited breaks during session to allow patients to attend to their needs.

The availability of evidence for remotely administered CBT is a significant development in the field of PD treatment. As noted by the authors of the largest study of face-to-face CBT in PD, practical considerations relating to attendance of appointments are the largest obstacle to patients accessing therapy: a fifth of their sample needed special arrangements to attend therapy visits while physical disability was cited as the most common reason for study exclusion. ${ }^{36}$ Remotely administered psychotherapy addresses these issues and has good evidence for efficacy in the treatment of depression in the general population. ${ }^{50}$ Its limitations are the potential for lower level of involvement with therapy - Kraepelien et a ${ }^{38}$ noted that in the feedback following their internetbased intervention participants admitted to not working actively with the assessments and that they did not readily contact the therapist when they had questions. These may, however, be issues specific to the internet-based format as Dobkin et $a l^{36}$ reported excellent retention rates for their telephone-administered CBT programme.

Several studies examined the effect of CBT-informed interventions on levels of carer burden and the results were not uniform. In general, where the primary study focus was improvement of neuropsychiatric symptoms in patients, a small number of carer education sessions were provided with the aim of carers facilitating the patients' practice of CBT technique. Carer burden was explored as secondary measure and the authors in general found no effect of the intervention on carer burden (eg, Dobkin et $\mathrm{a}^{25}$ controlled trial). In contrast, two independent groups have shown that CBT-informed programmes that target specifically PD carer burden are beneficial in a general sample of PD carers ${ }^{42}$ as well as in those experiencing significant psychological distress. ${ }^{46}$ These data suggest that treatment of PD carer is most likely to be effective where systematic CBT is applied.

Limited evidence exists also for targeted treatment of insomnia and ICD. Two small studies reported beneficial effect to sleep quality from CBT using face-to-face and telephone CBT. The second largest evaluation of CBT in PD focused on ICD and found good evidence for improvement in impulse control behaviours versus waiting list. A smaller study explored CBT efficacy for pathological gambling but was limited by its retrospective and small sample size that was recruited from a convenience population (clinic patients).

\section{Limitations}

Many of the studies included in this review are small and speculative, and essentially present pilot data. The conduct of many of the constituent studies is poor, with incomplete attention paid to participation rates, gaining representative samples of consecutive patients (instead of convenience samples) and small sample sizes. The reporting of many of the studies is also poor, with insufficient attention paid to descriptions of samples, poor reporting of 'negative' associations and failure to report effect sizes. Follow-up periods tended to be brief which limits the extent to which conclusions can be drawn about the longterm efficacy of CBT in PD. Furthermore, concomitant antidepressant and dopaminergic medication use was either significant or unreported, which suggests a potential confound. Nonetheless, there is a small body of better quality studies, and for some of the main findings there is consistency of results within the literature. Finally, depression/anxiety, ICD and insomnia do not exhaust the range of neuropsychiatric symptoms in PD-psychosis and apathy are prominent, significant and undertreated symptoms of the disease. We chose not to focus on these domains in our review on account of the limited existing evidence for their treatment with CBT.

\section{Conclusion}

Psychiatric manifestations in PD are a highly prevalent component of the disease that is a significant source of disability and carer burden. Psychopharmacological approaches are limited in the extent to which they can consistently ameliorate them. In this review paper, we found that there is a growing evidence base for non-pharmacological treatment of psychiatric symptoms with moderate effect sizes reported for the efficacy of CBT on depression, including where distance administration was used. In addition, there is some initial promising data on the effects of CBT on impulse-control disorders and insomnia. There is a need for adequately powered studies to strengthen the position of CBT as a viable treatment option for psychiatric symptoms in PD.

Competing interests None declared.

Provenance and peer review Not commissioned; externally peer reviewed. doi:10.1136/eb-2016-102574

Received 1 December 2016; Accepted 23 December 2016 


\section{REFERENCES}

1. Weintraub D, Burn DJ. Parkinson's disease: the quintessential neuropsychiatric disorder. Mov Disord 2011;26:1022-31.

2. Kulisevsky J, Pagonabarraga J, Pascual-Sedano B, et al. Prevalence and correlates of neuropsychiatric symptoms in Parkinson's disease without dementia. Mov Disord 2008;23:1889-96.

3. Forsaa EB, Larsen JP, Wentzel-Larsen T, et al. Predictors and course of health-related quality of life in Parkinson's disease. Mov Disord 2008;23:1420-7.

4. Landau S, Harris V, Burn DJ, et al. Anxiety and anxious-depression in Parkinson's disease over a 4-year period: a latent transition analysis. Psychol Med 2016;46:657-67.

5. Carod-Artal FJ, Ziomkowski S, MourãfO Mesquita $\mathrm{H}$, et al. Anxiety and depression: main determinants of health-related quality of life in Brazilian patients with Parkinson's disease. Parkinsonism Relat Disord 2008:14:102-8.

6. Hely MA, Morris JG, Reid WG, et al. Sydney multicenter study of Parkinson's disease: non-L-DOPA-responsive problems dominate at 15 years. Mov Disord 2005;20:190-9.

7. Weintraub D, Koester J, Potenza MN, et al. Impulse control disorders in Parkinson disease: a cross-sectional study of 3090 patients. Arch Neurol 2010;67:589-95.

8. Leroi I, Harbishettar V, Andrews $M$, et al. Carer burden in apathy and impulse control disorders in Parkinson's disease. Int J Geriatr Psychiatry 2012;27:160-6.

9. Dauvilliers Y. Insomnia in patients with neurodegenerative conditions. Sleep Med 2007;8(Suppl 4):S27-34.

10. Adler CH, Thorpy MJ. Sleep issues in Parkinson's disease. Neurology 2005;64 (Suppl 3):S12-20.

11. Stavitsky K, Neargarder S, Bogdanova Y, et al. The impact of sleep quality on cognitive functioning in Parkinson's disease. J Int Neuropsychol Soc 2012;18:108-17.

12. Troeung L, Egan SJ, Gasson N. A meta-analysis of randomised placebo-controlled treatment trials for depression and anxiety in Parkinson's disease. PLOS ONE 2013;8:e79510.

13. Menza $\mathbf{M}$, Dobkin RD, Marin $\mathrm{H}$, et al. The impact of treatment of depression on quality of life, disability and relapse in patients with Parkinson's disease. Mov Disord 2009;24:1325-32.

14. Moonen AJ, Wijers A, Leentiens AF, et al. Severity of depression and anxiety are predictors of response to antidepressant treatment in Parkinson's disease. Parkinsonism Relat Disord 2014;20:644-6.

15. Okai D, Samuel M, Askey-Jones S, et al. Impulse control disorders and dopamine dysregulation in Parkinson's disease: a broader conceptual framework. Eur J Neurol 2011;18:1379-83.

16. Lie JD, Tu KN, Shen DD, et al. Pharmacological treatment of insomnia. P T 2015; 40:759-71.

17. Braak H, Del Tredici K, Rüb U, et al. Staging of brain pathology related to sporadic Parkinson's disease. Neurobiol Aging 2003:24:197-211.

18. Pal PK, Calne S, Samii A, et al. A review of normal sleep and its disturbances in Parkinson's disease. Parkinsonism Relat Disord 1999;5:1-17.

19. Hofmann SG, Smits JA. Cognitive-behavioral therapy for adult anxiety disorders: a meta-analysis of randomized placebo-controlled trials. J Clin Psychiatry 2008;69:621-32

20. Simpson HB, Foa EB, Liebowitz MR, et al. A randomized, controlled trial of cognitive-behavioral therapy for augmenting pharmacotherapy in obsessive-compulsive disorder. Am J Psychiatry 2008;165:621-30.

21. Knott S, Woodward D, Hoefkens A, et al. Cognitive behaviour therapy for bulimia nervosa and eating disorders not otherwise specified: translation from randomized controlled trial to a clinical setting. Behav Cogn Psychother 2015:43:641-54.

22. Piacentini J, Woods DW, Scahill L, et al. Behavior therapy for children with Tourette disorder: a randomized controlled trial. JAMA 2010;303:1929-37.

23. Mohr DC, Likosky W, Bertagnolli A, et al. Telephone-administered cognitive-behavioral therapy for the treatment of depressive symptoms in multiple sclerosis. J Consult Clin Psychol 2000;68:356-61.

24. Kunik ME, Veazey C, Cully JA, et al. COPD education and cognitive behaviora therapy group treatment for clinically significant symptoms of depression and anxiety in COPD patients: a randomized controlled trial. Psychol Med 2008;38:385-96.

25. Dobkin RD, Menza M, Allen LA, et al. Cognitive-behavioral therapy for depression in Parkinson's disease: a randomized, controlled trial. Am J Psychiatry 2011:168:1066-74.
26. Dreissig H. Psychologic effects of structured cognitive psychotherapy in young patients with Parkinson disease: a pilot study. Nord J Psychiatry 1999;53:217-21.

27. Okai D, Askey-Jones S, Samuel M, et al. Trial of CBT for impulse control behaviors affecting Parkinson patients and their caregivers. Neurology 2013;80:792-9.

28. Rios Romenets S, Creti L, Fichten C, et al. Doxepin and cognitive behavioural therapy for insomnia in patients with Parkinson's disease - a randomized study. Parkinsonism Relat Disord 2013;19:670-5.

29. Troeung L, Egan SJ, Gasson N. A waitlist-controlled trial of group cognitive behavioural therapy for depression and anxiety in Parkinson's disease. BMC Psychiatry 2014;14:19.

30. Dobkin RD, Allen LA, Menza M. Cognitive-behavioral therapy for depression in Parkinson's disease: a pilot study. Mov Disord 2007:22:946-52.

31. Farabaugh A, Locascio JJ, Yap L, et al. Cognitive-behavioral therapy for patients with Parkinson's disease and comorbid major depressive disorder. Psychosomatics 2010;51:124-9.

32. Feeney F, Egan SJ, Gasson N. Treatment of depression and anxiety in Parkinson's Disease: a pilot study using group cognitive behavioural therapy. Clin Psychol 2005; 9:31-8.

33. Shinmei I, Kobayashi $\mathrm{K}, \mathrm{Oe} \mathrm{Y}$, et al. Cognitive behavioral therapy for depression in Japanese Parkinson's disease patients: a pilot study. Neuropsychiatr Dis Treat 2016:12:1319-31.

34. Cole K, Vaughan FL. The feasibility of using cognitive behaviour therapy for depression associated with Parkinson's disease: a literature review. Parkinsonism Relat Disord 2005;11:269-76.

35. Dobkin RD, Allen LA, Menza M. A cognitive-behavioral treatment package for depression in Parkinson's disease. Psychosomatics 2006;47:259-63.

36. Dobkin RD, Menza M, Allen LA, et al. Telephone-based cognitive-behavioral therapy for depression in Parkinson disease. J Geriatr Psychiatry Neurol 2011;24:206-14.

37. Veazey C, Cook KF, Stanley M, et al. Telephone-administered cognitive behavioral therapy: a case study of anxiety and depression in Parkinson's disease. J Clin Psychol Med Settings 2009;16:243-53.

38. Kraepelien M, Svenningsson P, Lindefors N, et al. Internet-based cognitive behavioral therapy for depression and anxiety in Parkinson's disease-a pilot study. Internet Interv 2015;2:1-6.

39. Dobkin RD. The relationship between telephone-administered cognitive-behaviora therapy for depression and neuropsychological functioning in Parkinson's disease. J Neuropsychiatry Clin Neurosci 2014;26:E10-11.

40. Hedman $\mathbf{E}$, Ljótsson B, Kaldo V, et al. Effectiveness of Internet-based cognitive behaviour therapy for depression in routine psychiatric care. J Affect Disord 2014;155:49-58

41. Watkins-Bruner D, Scott C, Lawton C, et al. RTOG's first quality of life studyRTOG 90-20: a phase II trial of external beam radiation with etanidazole for locally advanced prostate cancer. Int J Radiat Oncol Biol Phys 1995;33:901-6.

42. A'Campo LE, Spliethoff-Kamminga NG, Macht M, et al. Caregiver education in Parkinson's disease: formative evaluation of a standardized program in seven European countries. Qual Life Res 2010;19:55-64.

43. Macht M, Gerlich C, Ellgring H, et al. Patient education in Parkinson's disease: formative evaluation of a standardized programme in seven European countries. Patient Educ Couns 2007:65:245-52.

44. Simons G, Thompson SBN, Smith Pasqualini MC. An innovative education programme for people with Parkinson's disease and their carers. Parkinsonism Relat Disord 2006:12:478-85

45. Goldberg D. General health questionnaire. Windsor: NFER-Nelson, 1978.

46. Secker DL, Brown RG. Cognitive behavioural therapy (CBT) for carers of patients with Parkinson's disease: a preliminary randomised controlled trial. J Neurol Neurosurg Psychiatr 2005:76:491-7.

47. Jimenez-Murcia S, Bove Fl, Israel M, et al. Cognitive-behavioral therapy for pathological gambling in Parkinson's disease: a pilot controlled study. Eur Addict Res 2012;18:265-74

48. Lesieur HR, Blume SB. The South Oaks Gambling Screen (SOGS): a new instrument for the identification of pathological gamblers. Am J Psychiatry 1987;144:1184-8.

49. Yang $\mathbf{H}$, Petrini M. Effect of cognitive behavior therapy on sleep disorder in Parkinson's disease in China: a pilot study. Nurs Health Sci 2012;14:458-63.

50. Mohr DC, Vella L, Hart S, et al. The effect of telephone-administered psychotherapy on symptoms of depression and attrition: a meta-analysis. Clin Psychol (New York) 2008:15:243-53. 